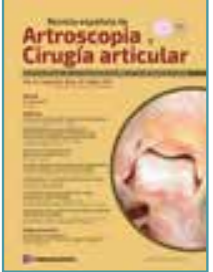

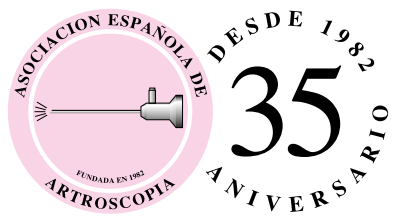

Vol. 24. Especial. Núm. 57. Mayo 2017

ISSN: 2386-3129 (impreso)

2443-9754 (online)

\title{
Original
}

\section{La salud del equipo quirúrgico en cirugía ortopédica y traumatología}

\section{J.A. Fernández Valencia', J.M. Rodríguez Roiz², V.A. Rodríguez Roiz M. Vives ${ }^{1}$, M. Orench ${ }^{4}$, S. Sastre ${ }^{1}$, A. Combalía'}

1 Servicio de Cirugía Ortopédica y Traumatología. Hospital Clínic. Universidad de Barcelona

2 Servicio de Cirugía Ortopédica y Traumatología. Mutua Fraternidad. Barcelona

${ }^{3}$ Servicio de Cirugía Ortopédica y Traumatología. Hospital de l'Esperit Sant. Santa Coloma de Gramenet

${ }^{4}$ Enfermería. Área Quirúrgica. Hospital Clínic. Universidad de Barcelona

Correspondencia:

Dr. Jenaro A. Fernández Valencia

Correo electrónico: jenarofv@clinic.ub.es
Recibido el 18 de marzo de 2017

Aceptado el 5 de abril de 2017

Disponible en Internet: mayo 2017

\section{RESUMEN}

Un equipo quirúrgico incluye a diferentes profesionales tales como cirujanos, anestesistas, instrumentistas, enfermería circulante o técnicos de radiodiagnóstico. Este equipo multidisciplinar, descrito en su funcionamiento óptimo como una "máquina bien engrasada", tiene como objetivo realizar las cirugias maximizando la seguridad del paciente y su satisfacción, y aumentando la calidad de los resultados. De esta forma, también se reducen de forma indirecta los costes en relación con las estancias hospitalarias y las complicaciones. En el desempeño de su trabajo, el equipo se enfrenta a diferentes factores de riesgo que pueden mermar su bienestar como grupo o bien la salud física o psíquica de cada uno de los miembros. En el presente artículo revisamos los principales factores que pueden poner en riesgo la salud del equipo quirúrgico en la especialidad de Cirugía Ortopédica y Traumatología, y cómo evitarlos o bien cómo minimizar su impacto.

Palabras clave: Riesgo laboral. Quirófano. Radiaciones. Infección. Daño ocupacional. Urolitiasis. Hábitos dietéticos.

\begin{abstract}
The health of the surgical team in orthopaedic surgery and traumatology
\end{abstract}

A surgical team includes different professionals such as surgeons, anesthetists, instrumentalists, circulating nursing or radiodiagnostic technicians. This multidisciplinary team, described in its optimal functioning as a "well-oiled machine", aims to perform the surgeries maximizing the patient safety and its satisfaction, while increasing the quality of the results. In this way, indirect costs are also reduced in relation to hospital stays and complications. In the performance of their work, the team faces different risk factors that can deplete its well-being as a group or affect the physical health of each one of its members. In the present article we make a review of the main factors that can put at risk the surgical team's health in the specialty of orthopedic surgery and traumatology, and how to avoid them, or how to minimize their impact.

Key words: Occupational risk. Surgery. Radiation. Infection. Occupational damage. Urolithiasis. Dietary habits. 


\section{Introducción}

En todo ejercicio profesional existen riesgos. Entre los profesionales con más riesgo se citan, obviamente, los militares, bomberos, policías o los trabajadores de la construcción ${ }^{(1)}$. En estas profesiones es frecuente el uso de dispositivos de protección, tales como cascos, chaleco antibalas o protección auditiva y/o visual. Si bien el ejercicio de la cirugía ortopédica supone un riesgo menor para la salud, consideramos que posiblemente la sensibilización respecto de los riesgos es baja. Este artículo pretende repasar los riesgos para la salud del equipo quirúrgico y cómo evitarlos o minimizarlos.

Repasaremos cómo nos afectan y cómo protegernos de radiaciones ionizantes, humo quirúrgico, enfermedades infecto-contagiosas, ruido y productos químicos. Finalmente, abordaremos cuestiones sobre la nutrición y la hidratación. A pesar de no poder tratarse cada uno de los temas con la adecuada profundidad, esperamos que el lector encuentre interesante su lectura y que incluso pueda sacar algunas conclusiones para su práctica diaria que puedan revertir en una mejora de su salud actual o futura.

\section{Las radiaciones ionizantes}

En Cirugía Ortopédica y Traumatología se sigue necesitando del uso de radiaciones ionizantes durante la cirugía (Figura 1). Los efectos de las radiaciones se engloban en 2 categorías: los dependientes de la dosis recibida, denominados efectos no estocásticos, y los independientes de la dosis, denominados efectos estocásticos. Los efectos no estocásticos se pueden evitar si no pasamos de una determinada dosis para un determinado efecto. En cambio, los efectos estocásticos no tienen una dosis límite y pueden acabar traduciéndose en una neoplasia después de un periodo latente que puede durar de años a décadas dependiendo del tipo de cáncer ${ }^{(2)}$. No es el objetivo de este apartado repasar todos conceptos básicos de protección y seguridad radiológica(3). Sin embargo, vemos interesante reseñar algunos hallazgos, con el objetivo de sensibilizar en la maximización de la protección respecto a las radiaciones ionizantes. Por un lado, se ha constatado mayor incidencia de patología oncológica

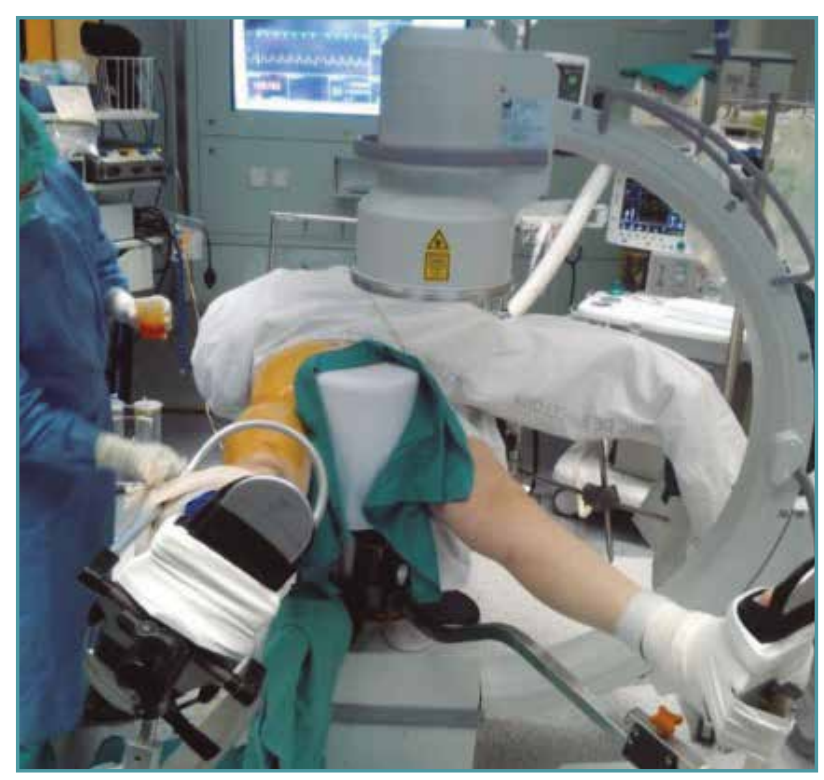

Figura 1. Exposición a radiaciones ionizantes. Se debe minimizar el uso de radiaciones ionizantes, alejarse en lo posible del arco y llevar protección adecuada.

en Cirugía Ortopédica y Traumatología respecto de otras especialidades. En un estudio realizado por Mastrangelo et al.(4) la incidencia acumulada de cáncer para el periodo de 1976 a 2000 fue del $29 \%$ (9/31) en cirugía ortopédica, del 6\% (8/125) en otros profesionales expuestos a radiaciones ionizantes, pero no en cirugía ortopédica, y del $4 \%(7 / 158)$ en trabajadores no expuestos. Se efectuó un análisis de regresión logística que mostró que los cirujanos ortopédicos tienen un riesgo aumentado de desarrollo de tumores $(p<0,002)$. Recientemente, ha cobrado interés que, en las mujeres en el ejercicio de la cirugía ortopédica, existe un mayor riesgo de desarrollo de cáncer de mama respecto a la población general ${ }^{(5)}$. De forma similar, los técnicos de radiodiagnóstico también tienen incidencias más elevadas de tumores. Por otro lado, otra de las posibles complicaciones al exponernos a las radiaciones ionizantes es el desarrollo de cataratas. Ya existe literatura con seguimiento a 20 años que indica el aumento de la incidencia de cataratas en técnicos de radiodiagnóstico, específicamente de tipo subcapsular(6). Al parecer, existe controversia respecto a la dosis necesaria para el desarrollo de cataratas, pero si se consideraba que existía un efecto determinista a partir de 5 a 8 Gy, actualmente se ha bajado el nivel a 0,5 Gy. 
Finalmente, e importante para aquellos que nos dedicamos a la docencia, la cantidad de radiación que recibimos es inversamente proporcional a la experiencia quirúrgica y a la complejidad de la cirugía a tratar ${ }^{(7)}$. Este es un motivo más para enfrentar al residente de forma progresiva a problemas cada vez más complejos.

Para minimizar el riesgo de complicaciones en relación con las radiaciones ionizantes, debemos reducir su uso al mínimo necesario, alejarnos en lo posible del aparato de rayos $X$ y del paciente, $y$ llevar las medidas de protección adecuadas. Entre las medidas de protección, se usan los delantales de plomo que cubren tórax, pelvis y muslo proximal y se infravalora en ocasiones el uso del protector tiroideo. Sería recomendable también usarlo, al igual que las gafas con cristal ploma$\mathrm{do}^{(8)}$. Respecto al uso de guantes plomados, se ha constatado que reducen del 7 al 50\% la dosis recibida, pero se ha criticado que pueden proporcionar una falsa seguridad que incitaría a un mayor uso de rayos $X^{(9)}$.

\section{Efectos del humo quirúrgico}

El humo quirúrgico procede de la utilización de instrumentos electroquirúrgicos para el corte y la cauterización de los tejidos y contiene pequeñas partículas consideradas potencialmente nocivas. Se trata de un $80 \%$ de vapor de agua con un $20 \%$ de sustancias: formaldehído (irritante y carcinógeno potencial), acetaldehído (carcinógeno), benceno (carcinógeno) y tolueno (irritante de vías respiratorias y ojos al igual que neurotóxico)(10).

Se recomienda que los quirófanos experimenten un mínimo de 15 a 20 renovaciones del aire por hora y que la presión dentro de ellos sea positiva respecto a la de su entorno. Esta medida contribuye a eliminar gases anestésicos y otros productos, tales como el humo quirúrgico, que puedan acumularse en la sala quirúrgica. Sin embargo, la renovación de aire no ha demostrado ser suficiente para disminuir la exposición al humo quirúrgico, al igual que tampoco protegen suficientemente las máscaras quirúrgicas convencionales ${ }^{(11)}$. Se han desarrollado aspiradores especialmente diseñados para extraer el humo quirúrgico en el mismo campo quirúrgico, pero su eficacia todavía está por ser demostrada. Consideramos que el humo quirúrgico supone uno de los riesgos menos conocidos y más infravalorados para el equipo quirúrgico en nuestra especialidad y merece mayor estudio en el futuro.

\section{Transmisión de infecciones por punción o salpicadura}

La principal vía de exposición es por punción accidental durante la cirugía (con fragmentos de hueso o material quirúrgico punzante) o bien por salpicadura sobre una mucosa, en especial sobre la conjuntiva. Los virus más frecuentes son los virus de la hepatitis B y C (VHB y VHC) y el virus de la inmunodeficiencia humana (VIH). Los riesgos estimados de contraer una infección a través de una lesión de la piel son del 0,3\% para el VIH, del 6 al 30\% para el VHB y del 1,8\% para el $\mathrm{VHC}^{(9)}$. Según información de 2013 del Centro de Control y Prevención de Enfermedades (CDC), se han confirmado 57 casos de seroconversión a VIH en trabajadores de la salud después de una exposición ocupacional de riesgo alto para VIH y 143 casos de posible transmisión en pacientes sin otro factor de riesgo conocido.

Los profesionales con accidente de riesgo biológico deben seguirse por 6 meses. Se recomiendan controles en el momento del accidente, a la sexta semana, al tercer mes y al sexto mes tras la exposición. En el momento del accidente tanto la fuente como el trabajador accidentado deben evaluarse para VIH, VHC, VHB y, si hay antecedentes de vacunación para VHB en el trabajador accidentado, realizar determinación de anticuerpos contra el AgsHB (Acs contra AgsHB) ${ }^{(12)}$. El resultado de los exámenes no debe retrasar el inicio de la terapia de profilaxis si se considera fuente de alto riesgo por el interrogatorio o la fuente se niega a realizarse exámenes. En aquellos con indicación de profilaxis, esta debe iniciarse en las primeras horas del accidente, idealmente menos de $24 \mathrm{~h}$ y máximo hasta 72 h. Después de este tiempo, no se ha demostrado efectividad en su inicio.

Una medida extendida para intentar disminuir el riesgo de punción accidental es usar doble guante. En un metaanálisis reciente de Cochrane, se concluye que existe evidencia de calidad moderada que soporta el uso de doble guante respecto a solo uno, al evitar las lesiones cutáneas con punciones intraoperatorias ${ }^{(13)}$. También aumenta el riego de contagio si nos accidentamos 


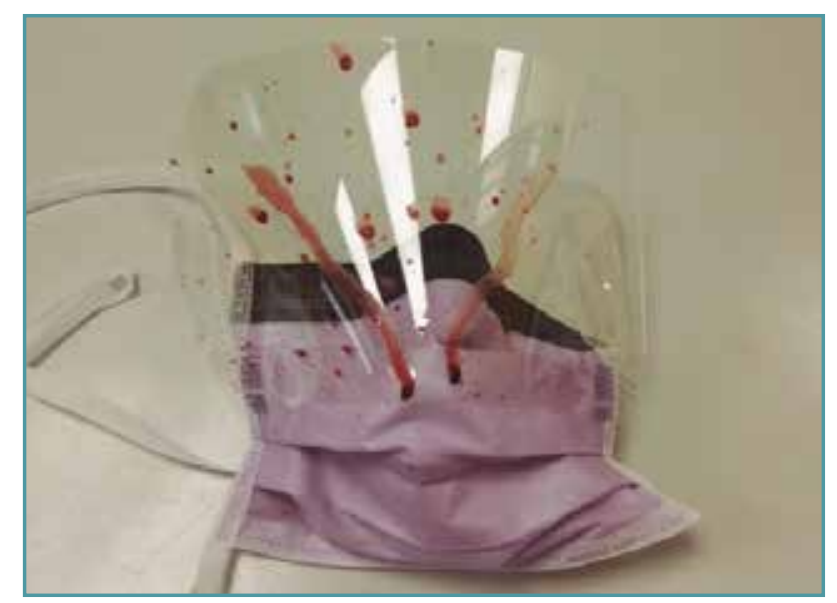

Figura 2. Salpicaduras en mascarilla quirúrgica tras una artroplastia de cadera. Máscara de cirujano principal. Aunque no ocurre asi en absoluto en todas las cirugías, en ocasiones el sangrado de un vaso o la impactación de un componente puede salpicar la cara del cirujano con esta contundencia.

con un material punzante y canulado (aguja intramuscular), que con un material simplemente punzante no canulado (aguja de Kirschner).

En un estudio realizado por Demircay et al.(14), se identificó la perforación de guante externo en el $18,4 \%$ de los casos y del guante interno en el $8,4 \%$ de los casos. Curiosamente, el lugar más frecuente de perforación fue el segundo dedo de la mano no dominante y la mayoría de las perforaciones ocurrieron durante la primera mitad del procedimiento.

Algo frecuente y menos conocido es el atrapamiento accidental del guante con un sistema a motor giratorio. En estos casos, a pesar de que macroscópicamente el guante no aparente ruptura, un estudio reciente indica que existen frecuentemente microperforaciones no valorables a simple vista(15).

Respecto a la transmisión de infecciones por salpicadura, puede ocurrir por el sangrado imprevisto de un vaso sanguíneo o bien durante diferentes tiempos de la cirugía, que implican el raspado de material en el interior del hueso, el uso de sierras oscilantes o la impactación de implantes (Figura 2). El material salpicado puede ser sangre, grasa o incluso fragmentos de hueso y/o cemento (en el caso de recambios protésicos).

Respecto a la sangre salpicada, no todas las especialidades quirúrgicas tienen el mismo riesgo de recibir impactos en la región facial y cervical. Se ha documentado que la especialidad con más riesgo es la cirugía cardiovascular, seguida de la neurocirugía, la cirugía gastrointestinal y la cirugía ortopédica ${ }^{(16)}$. Tal y como documentaron Alani et al. ${ }^{(17)}$, tanto en artroplastia de rodilla como en artroplastia de cadera existe un elevado riesgo de salpicadura. En su estudio evaluaron las salpicaduras en la región facial del cirujano y el ayudante, expresando en cifras los impactos en 3 regiones: boca/labios, nariz/ mejillas y región ocular. La media de impactos en artroplastia de rodilla (sobre 11 cirugías realizadas) en boca/labios, nariz/mejillas y región ocular fue de 217, 105 y 62 para el cirujano y de 258, 147 y 82 para el ayudante, respectivamente. La media de impactos en artroplastia de cadera (sobre 14 cirugías realizadas) en boca/labios, nariz/mejillas y región ocular fue de 214, 90 y 53 para el cirujano y de 137, 39 y 27 para el ayudante, respectivamente.

Estos resultados contrastan con un estudio publicado por Nagao et al. ${ }^{(18)}$, en el que el profesional que más riesgo biológico declaraba por salpicadura era el/la instrumentista. Este estudio se trataba de una revisión retrospectiva de 7 años en un hospital universitario. De forma interesante, solo un $22 \%$ de los facultativos reportó todos los episodios de exposición de riesgo biológico. Da la impresión, en función de este y otros estudios, de que existe de forma generalizada una infravaloración del riesgo de transmisión de enfermedades infecciosas por salpicadura. Muchos cirujanos optan por no llevar protección ocular o bien por llevar sus gafas habituales. Respecto a este segundo grupo, es interesante que revisen el estudio por Mansour et al.(15), en el que demostraron que llevando gafas convencionales no se tiene una protección significativamente superior respecto a los que no las llevan. Se deberían utilizar gafas de protección amplia, similares a las utilizadas en la industria de la construcción, o bien máscaras quirúrgicas con pantalla de protección frontal y lateral.

Como resumen respecto a la adquisición de enfermedades infecciosas, las medidas de protección se centrarán en considerar al paciente siempre como potencialmente infeccioso, usar guantes durante la colocación del paciente y usar doble guante y protección ocular específica durante la cirugía ${ }^{(19)}$. 


\section{Ruido quirúrgico}

Se ha llegado a documentar que el $50 \%$ de los traumatólogos presenta signos de disminución de audición secundaria a la exposición a traumatismo acústico crónico ${ }^{(20)}$. Se considera que la exposición al ruido es peligrosa por encima de $85 \mathrm{~dB}$ durante 8 horas y en algunos estudios se ha cuantificado el ruido en un quirófano de traumatólogos entre 74,8 y $82,1 \mathrm{~dB}$ de ruido medio en artroplastia de cadera y rodilla; entre 88 y $142 \mathrm{~dB}$ con diferentes instrumentales en el oído del cirujano; y entre 71 y 96 dB a 2 metros de él. Algunos modelos de sierras y motores producen niveles de entre 90 y $100 \mathrm{~dB}$ en el oído del cirujano y de 80 a 90 dB a 3 metros de él. Estos últimos niveles son los más preocupantes, porque a mayores decibelios, mayor riesgo de lesiones permanentes. Por ejemplo, en el caso de pasar de $110 \mathrm{~dB}$ por más de 1 minuto de forma frecuente, se produce una pérdida de audición de forma definitiva ${ }^{(9)}$.

Se ha evaluado el ruido al nivel del pabellón auditivo del cirujano con el uso de trajes espaciales para la cirugía: con el ventilador puesto, la media fue de $61 \mathrm{~dB}$, aproximadamente el nivel conversacional. Sin embargo, esto crea dificultades para poder hablar con el resto del equipo ${ }^{(21)}$. Este fenómeno de interferencia de la protección auditiva con la comunicación es bien conocido en la industria aeronáutica (como ocurre con los pilotos de helicópteros) y todavía está en proceso de mejora.

En función de estos hallazgos, se recomendaría a los cirujanos e instrumentistas especializados en cirugía ortopédica y traumatología que pasen revisiones regulares de audición, y se recomienda que usen protección auditiva sobre todo al usar algunos instrumentales. También se recomienda a la industria investigar en el desarrollo de instrumental menos ruidoso y en sistemas de protección con comunicación entre los miembros del equipo quirúrgico.

Por otro lado, y según nuestro conocimiento no estudiado en la literatura, se debería evaluar la exposición de anestesistas y su posible necesidad de protección auditiva. Al estar expuestos de forma repetida a múltiples procedimientos potencialmente nocivos para su audición, puede que también fuese recomendable para ellos el uso de tapones u orejeras. Aunque no suelen permanecer tanto tiempo seguido en quirófano como los traumatólogos, pasan más días en su interior.

\section{Productos químicos}

Comentamos en este apartado 2 cuestiones: la dermatitis de contacto y las reacciones al cemento. En cuanto a los guantes y al riesgo de dermatitis de contacto: se han descrito cifras de sensibilización al látex en el 1\% de la población y en cerca del 10 al 20\% del personal sanitario. Más de la mitad de los profesionales con problemas cutáneos provienen del ámbito sanitario, estando en especial riesgo los trabajadores de áreas quirúrgicas y especialistas dentales. Este aumento de riesgo está en relación con que gran cantidad de los materiales que se emplean actualmente en el ámbito sanitario están compuestos de agentes químicos que pueden afectar a la dermis y contienen látex, como son jeringas, sondas, catéteres y especialmente los guantes.

Existen personas alérgicas al látex y personas con hipersensibilidad al mismo, siendo la exposición continuada el principal factor de riesgo en la producción de sensibilización, por lo que el uso prolongado y repetido de este material provoca un aumento en la incidencia y la prevalencia de la dermatitis. Esta hipersensibilidad puede incrementar la gravedad de los sintomas durante la exposición repetida al agente causante, incluso pueden aparecer reacciones cruzadas con algunos alimentos, como ocurre en el denominado sindrome de látex-frutas.

Hay que diferenciar las reacciones adversas al látex entre inmunológicas y no inmunológicas. Las reacciones no inmunológicas desencadenan la dermatitis de contacto (Figura 3), manifestada como una reacción eccematosa con prurito, frecuente en el dorso de la mano y en los dedos: se considera una irritación "mecánica" o por el pH alcalino de los guantes, pero independiente de la composición química de los guantes ${ }^{(22)}$. En este contexto, los guantes sin polvo son un gran aliado. Las reacciones inmunológicas suponen mayor complejidad, existen hasta 15 alérgenos descritos responsables de estas reacciones y se escapan del objetivo de esta revisión.

La segunda cuestión es el cemento. Es uno de los productos químicos más utilizados en cirugía ortopédica y traumatología y se basa en el polimetil-metacrilato (PMMA). Se han descrito efectos tóxicos ocasionados por el PMMA para el tracto respiratorio, el sistema neurológico y la piel ${ }^{(23,24)}$. Se han publicado múltiples casos de irritación 


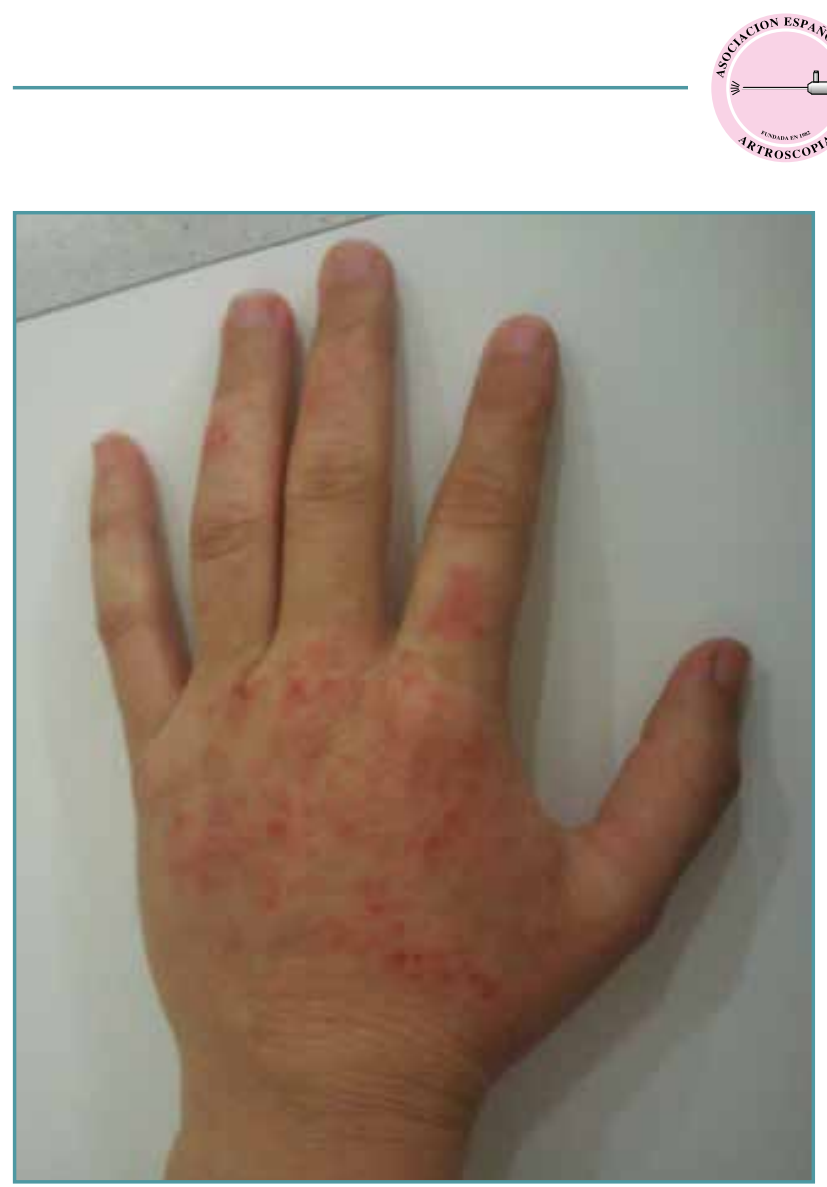

Figura 3. Imagen clínica de dermatitis de contacto de cirujano en mano izquierda. Se aprecian lesiones características localizadas en el dorso de la mano y los dedos.

ocular, nasal y del tracto respiratorio superior en relación con picos de exposición de corto tiempo y en la literatura se han descrito un total de 19 casos más graves que incluyen asma, laringitis o neumonitis por hipersensibilidad ${ }^{(25)}$. Una de las recomendaciones es que la mezcla del cemento debería realizarse en sistemas de vacío porque disminuye la porosidad del mismo, mejorando sus propiedades mecánicas, pero también porque disminuye la exposición del personal de quirófano. Por otro lado, no se recomienda masajear o toquetear el cemento con los dedos para ir comprobando el fraguado.

\section{Hidratación}

Probablemente, no nos hidratamos adecuadamente. En un estudio realizado en la Mayo Clinic, se evaluó la prevalencia de urolitiasis entre 3.900 de sus empleados. En general, la prevalencia fue del 10,9\%; el personal de quirófano llegó a una prevalencia del $14,6 \%$ y los cirujanos a un $17,6 \%$, siendo estas diferencias significativas. El equipo quirúrgico tiene mayor riesgo de desarrollo de urolitiasis, reconociéndose que trabaja en condiciones de mayor estrés y también porque de forma significativa reportaron una menor toma de líquidos ${ }^{(26)}$. Sería recomendable que no desoigamos a nuestro cuerpo; en especial, con cirugías largas o en las que "hemos sudado la camiseta", y que tengamos por costumbre beber frecuentemente bien agua o incluso bebidas isotónicas, dependiendo de las pérdidas. Por otro lado, en los últimos años se han introducido los sistemas de mantas de aire forzado para mantener la normotermia del paciente. Este mantenimiento de la normotermia tiene grandes beneficios tanto fisiológicos como psicológicos; hoy en día no se cuestiona $^{(27,28)}$. Sin embargo, no es infrecuente que los cirujanos nos quejemos del calor bajo el que trabajamos, al estar en directo contacto con estas mantas, que en ocasiones llegan a estar programadas a $36040{ }^{\circ} \mathrm{C}$. Si el ambiente de quirófano puede estar entre 19 y $21^{\circ} \mathrm{C}$, bajo las luces y al lado de la manta se ha llegado a cuantificar en $25^{\circ} \mathrm{C}$. Si se añade que la cirugía es una cirugía de alto consumo energético, con estrés y calor, junto con el uso de delantales de plomo, el resultado es la deshidratación del cirujano y el ayudante, sumando también el riesgo de la producción de sudor que puede caer en el campo quirúrgico, bien rebosando desde los guantes hacia las mangas del traje quirúrgico o bien como gota de sudor desde la frente del cirujano al campo quirúrgico. Este fenómeno no ha sido suficientemente descrito o estudiado. Consideramos que se debe mantener la normotermia del paciente y también el correcto ambiente de trabajo para el equipo quirúrgico.

Un reciente estudio apunta a que el mantenimiento de la normotermia se puede conseguir con mantas reflectivas con la misma seguridad que con los sistemas de aire forzado, asociando medidas de precalentamiento del paciente y el uso de sueros calentados(29); consideramos que probablemente este podría ser el camino para proporcionar al paciente la máxima seguridad en cuanto a normotermia y también al cirujano en cuanto al riesgo de deshidratación y confort durante la cirugía. Sería bueno realizar estudios desde la perspectiva del equipo quirúrgico para valorar el grado de impacto de las mantas de 
aire forzado u otras medidas de calor en el disconfort del cirujano y del resto del equipo. Por el momento, para aquellos que sufran los inconvenientes del calor de la manta de aire forzado, también insistimos en la hidratación abundante entre cirugías.

\section{Alimentación}

Este apartado no está fundamentado tanto en la literatura como en impresiones personales. Vemos con frecuencia que los miembros del equipo quirúrgico tienen dificultad para prever sus tiempos de descanso y programar su alimentación. A lo largo de una jornada, en especial con cirugías largas, algunos optan al acabar por comer algo de la máquina dispensadora, repleta de productos con hidratos de carbono y grasa. A este alimento se le añade el líquido obtenido en la máquina dispensadora vecina, que acostumbra a ser un café. Otros van al bar del hospital y, dado que ya han cerrado la cocina, se piden el bocadillo de tortilla o el de beicon con una lata de una bebida carbonatada y azucarada. No se come nada más a lo largo del día y, en ocasiones, estos profesionales encadenan su actividad con la guardia, con jornadas de prolongación horaria de tarde o bien trabajando en otro centro por la tarde. En esa otra actividad tampoco tienen margen para comer de una forma ordenada, pudiendo existir alguna que otra ingesta proveniente de la máquina dispensadora y, al llegar la noche, se produce un fenómeno de hipercompensación que ha llegado a denominarse dieta yoyó (mínima ingesta y máxima ingesta en el mismo día): la cena intenta reparar la falta de calorías y es copiosa. Esta descripción puede resultar anecdótica, pero pensamos que describe una situación que puede ser cotidiana para algunos. Estos hábitos predisponen al desarrollo de obesidad y, con ello, el aumento del riesgo cardiovascular. Sin ser los autores expertos en dietética, es fácil que se intuya que en estas dietas existe una carencia de alimentos básicos entre los que deberían estar las cinco piezas de fruta y/o verdura al día, y que se debería intentar conseguir una dieta ordenada.

Sharma et al. ${ }^{(30)}$ plantearon si los trabajadores de hospital tenían una vida saludable. Realizaron un estudio a 924 empleados en 6 hospitales en Texas y encontraron que el $78,1 \%$ tenía sobre- peso o era obeso. Los empleados obesos (definidos como índice de masa corporal de más de $30 \mathrm{~kg} / \mathrm{m}$ ) tenían como hábitos un mayor consumo de patatas, grasas, bebidas azucaradas y pasaban más tiempo viendo la televisión, jugando a videojuegos o sentándose que los empleados que tenían un peso normal. Sería interesante un estudio asi en nuestro medio.

Son muchos los factores que inciden en la salud presente y futura de un equipo quirúrgico. En esta revisión se han tratado aspectos concretos respecto de la actividad en cirugía ortopédica y traumatología. Si tuviéramos que resumirlo en una sola frase sería algo así como: "Usa doble guante, utiliza protección ocular adecuada contra las salpicaduras, cuida tu oído, minimiza tu exposición a radiaciones ionizantes, evita el humo quirúrgico, hidrátate bien y come saludable".

\section{Bibliografía}

1. News Release. Bureau of Labor Statistics. U.S. Department of Labor. December 2016. Disponible en: https:// www.bls.gov/news.release/pdf/cfoi.pdf.

2. Hynes DE, Conere T, Mee MB, Cashman WF. Ionising radiation and the orthopaedic surgeon. J Bone Joint Surg (Br). 1992;74-B:332-4.

3. Sarabia-Ribera GE. Protección y seguridad radiológicas. An Radiol Méx. 2013;2:105-10.

4. Mastrangelo G, Fedeli U, Fadda E, Giovanazzi A, Scoizzato L, Saia B. Increased cancer risk among surgeons in an orthopaedic hospital. Occup Med (Lond). 2005;55:498-500.

5. Valone LC, Chambers M, Lattanza L, James MA. Breast Radiation Exposure in Female Orthopaedic Surgeons. J Bone Joint Surg (Am). 2016;98-A:1808-13.

6. Chodick G, Bekiroglu N, Hauptmann M, Alexander BH, Freedman DM, Doody MM, et al. Risk of cataract after exposure to low doses of ionizing radiation: a 20-year prospective cohort study among US radiologic technologists. Am J Epidemiol. 2008;168:620-31.

7. Quah C, Mehta R, Shivji FS, Hassan S, Chandrasenan J, Moran CG, et al. The effect of surgical experience on the amount of radiation exposure from fluoroscopy during dynamic hip screw fixation. Ann R Coll Surg Engl. 2017;99:198-202.

8. Waddell BS, Waddell WH, Godoy G, Zavatsky JM. Comparison of Ocular Radiation Exposure Utilizing Three Types of Leaded Glasses. Spine (Phila Pa 1976). 2016;41:231-6. 


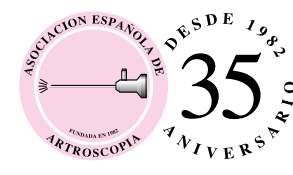

9. Lester JD, Hsu S, Ahmad CS. Occupational hazards facing orthopedic surgeons. Am J Orthop (Belle Mead NJ). 2012;41:132-9.

10. Al Sahaf OS, Vega-Carrascal I, Cunningham FO, McGrath JP, Bloomfield FJ. Chemical composition of smoke produced by high-frequency electrosurgery. Ir J Med Sci. 2007;176:229-32.

11. Romano F, Gustén J, De Antonellis S, Joppolo CM. Electrosurgical Smoke: Ultrafine Particle Measurements and Work Environment Quality in Different Operating Theatres. Int J Environ Res Public Health. 2017;14.

12. Kuhar DT, Henderson DK, Struble KA, Heneine W, Thomas V, Cheever LW, et al. Updated US Public Health Service guidelines for the management of occupational exposures to human immunodeficiency virus and recommendations for postexposure prophylaxis. Infect Control Hosp Epidemiol. 2013; 34:875-92.

13. Mischke C, Verbeek JH, Saarto A, Lavoie MC, Pahwa M, ljaz S. Gloves, extra gloves or special types of gloves for preventing percutaneous exposure injuries in healthcare personnel. Cochrane Database Syst Rev. 2014;(3):CD009573.

14. Demircay E, Unay K, Bilgili MG, Alataca G. Glove perforation in hip and knee arthroplasty. J Orthop Sci. 2010;15:790-4.

15. Goldman AH, Haug E, Owen JR, Wayne JS, Golladay G). High Risk of Surgical Glove Perforation From Surgical Rotatory Instruments. Clin Orthop Relat Res. 2016;474:2513-7.

16. Endo S, Kanemitsu K, Ishii H, Narita M, Nemoto T, Yaginuma $\mathrm{G}$, et al. Risk of facial splashes in four major surgical specialties in a multicentre study. J Hosp Infect. 2007;67:56-61.

17. Alani A, Modi C, Almedghio S, Mackie I. The risks of splash injury when using power tools during orthopaedic surgery: a prospective study. Acta Orthop Belg. 2008;74:678-82.

18. Nagao $M$, linuma $Y$, Igawa J, Matsumura $Y$, Shirano $M$, Matsushima A, et al. Accidental exposures to blood and body fluid in the operation room and the issue of underreporting. Am J Infect Control. 2009;37:541-4.

19. Wright JG, McGeer A. Human immunodeficiency virus transmission between surgeons and patients in orthopaedic surgery. Clin Orthop Relat Res. 1993;297:272-81.

20. Willett KM. Noise-induced hearing loss in orthopaedic staff. J Bone Joint Surg (Br). 1991;73-B:113-5.

21. Pearlman RT, Sandidge O. Noise characteristics of surgical space suits. Orthopedics. 2009;32:825.

22. Kahn SL, Podjasek JO, Dimitropoulos VA, Brown CW Jr. Natural rubber latex allergy. Dis Mon. 2016;62:5-17.

23. Leggat PA, Smith DR, Kedjarune U. Surgical applications of methyl methacrylate: a review of toxicity. Arch Environ Occup Health. 2009;64:207-12.

24. Parizi JL, Nai GA, Batalha CF, Lopes CC, Rizzo MF, Falcone $\mathrm{CE}$, et al. Assessment of methyl methacrylate vapor toxicity on the rat tracheal epithelium. Braz Oral Res. 2005;19:223-7.

25. Borak J, Fields C, Andrews LS, Pemberton MA. Methyl methacrylate and respiratory sensitization: a critical review. Crit Rev Toxicol. 2011;41:230-68.

26. Linder BJ, Rangel LJ, Krambeck AE. The effect of work location on urolithiasis in health care professionals. Urolithiasis. 2013;41:327-31.

27. Allen MW, Jacofsky DJ. Normothermia in Arthroplasty. J Arthroplasty. 2017. [Epub ahead of print]

28. Madrid E, Urrútia G, Roqué i Figuls M, Pardo-Hernandez $\mathrm{H}$, Campos JM, et al. Active body surface warming systems for preventing complications caused by inadvertent perioperative hypothermia in adults. Cochrane Database Syst Rev. 2016;4:CD009016.

29. Tjoakarfa C, David V, Ko A, Hau R. Reflective Blankets Are as Effective as Forced Air Warmers in Maintaining Patient Normothermia During Hip and Knee Arthroplasty Surgery. J Arthroplasty. 2017;32:624-7.

30. Sharma SV, Upadhyaya M, Karhade M, Baun WB, Perkison WB, Pompeii LA, et al. Are Hospital Workers Healthy?: A Study of Cardiometabolic, Behavioral, and Psychosocial Factors Associated With Obesity Among Hospital Workers. J Occup Environ Med. 2016;58:1231-8. 